\title{
EDITORIAL
}

\section{IN THE OCTOBER 2009 ISSUE OF CLINICS}

\author{
Mauricio Rocha-e-Silva, Editor
}

doi: $10.1590 / \mathrm{S} 1807-59322009001000001$

In this issue of CLINICS we highlight a report by Schout et al. on the epidemiological data relating to the pandemic novel influenza A (H1N1) infection which was considered widespread in Brazil on July 16, 2009. Since then, 46,810 cases have been reported in Brazil, most of them concentrated in São Paulo. Through September 16, 9,249 cases of novel influenza A H1N1in Brazil were confirmed, including 699 deaths. The mortality rate observed in Brazil is 0.47/100,000 inhabitants and varies according to the region. In this period, São Paulo registered 3733 cases (40.3\% of the total) of novel influenza A (H1N1) infection and 327 deaths, reflecting a mortality rate of $0.79 / 100,000$ inhabitants. The Hospital das Clínicas da Faculdade de Medicina da Universidade de São Paulo (HC) is a reference center for H1N1 cases in São Paulo. During the winter of 2009, 472 patients in this hospital were diagnosed with H1N1 infection; of these, 210 were admitted, and 16 died. To control this pandemic and to provide adequate care for these patients, the Hospital das Clínicas implemented "bundles" including prevention strategies, an epidemiologic surveillance service, availability of fast diagnosis, antiviral treatment and training of staff. A description of the epidemiologic features of this novel human influenza A (H1N1) infection in patients admitted to the Hospital is provided.

Clinics also publishes 9 papers on original research.

Lopes et al. evaluated serum alanine aminotransferase levels before and after a hemodialysis session and correlated these values with hematocrit rate and weight loss during the session in 146 patients with chronic renal failure. They describe significant elevations in the aminotransferase and hematocrit in parallel to a reduction in body weight after the hemodialysis session. They suggest that one of the factors for low alanine aminotransferase levels prior to hemodialysis could be hemodilution in patients with chronic renal failure.

Onakpoya et al. conducted a retrospective review of all (286) patients less than 15 years old who presented to the

Hospital das Clínicas, Faculdade de Medicina da Universidade de São Paulo - São Paulo/SP, Brazil.

mrsilva36@hcnet.usp.br eye clinic of Wesley Guild Hospital Ilesa, Nigeria between January 2001 and December 2006. They found that ocular trauma, allergic conjunctivitis, infections of the eye and its adnexa, and refractive errors were the most common conditions. They conclude that the prevalent childhood eye diseases can lead to absenteeism from school and are potentially blinding. Health education aimed at the prevention of ocular trauma and prompt presentation for the management of other eye diseases should be encouraged.

Santhiago et al. evaluated intraindividual visual acuity, wavefront errors and modulation transfer functions in patients implanted with two diffractive multifocal intraocular lenses in 40 eyes of 20 cataract patients who underwent phacoemulsification and implantation of a spherical multifocal ReSTOR intraocular lens in one eye and an aspheric Tecnis ZM900 multifocal intraocular lens in the other eye. Uncorrected photopic distance, near visual acuity and the defocus curve were measured, over a 3-month follow-up period. The wavefront error and modulation transfer function were also evaluated. They coclude that the ReSTOR SN60D3 and Tecnis ZM 900 intraocular lenses provided similar photopic visual acuity at distance and near. The diffractive intraocular lenses studied provided a low value of coma and spherical aberrations, with the Tecnis intraocular lens having a statistically lower spherical aberration compared to the ReSTOR intraocular lens. In the $5 \mathrm{~mm}$ pupil diameter analyses, both intraocular lens groups showed similar modulation transfer functions.

Alessi et al. present their experience in the treatment of 123 cutaneous tumors of various types, including basal and squamous cell carcinomas, Bowen's disease, erythroplasia of Queyrat, Paget's disease, and trichoepithelioma, with 5\% imiquimod cream from 2003 to 2008 and claim that their experience confirms imiquimod as an effective treatment option for several types of cutaneous tumors, especially in patients without cutaneous comorbidities

Ladeira et al. determined the accuracy of the MiniMental State Examination combined with the Verbal Fluency Test and Clock Drawing Test for the identifica- 
tion of patients with mild cognitive impairment $(n=83)$ and Alzheimer's disease $(n=81)$ compared to cognitively unimpaired controls $(n=83)$, based on clinical and neuropsychological data. They conclude that although they were accurate enough for the identification of Alzheimer's disease, neither test alone proved adequate for the correct separation of patients with mild cognitive impairment from healthy subjects. Combining these tests did not improve diagnostic accuracy, as compared to the Mini-Mental State Examination alone, in the identification of patients with mild cognitive impairment or Alzheimer's disease. Their data do not warrant the combined use of the Mini-Mental State Examination, the Verbal Fluency Test and the Clock Drawing Test as a sufficient diagnostic schedule in screening for mild cognitive impairment.

Pereira et al. endeavored to develop a pointing device controlled by head movement that had the same functions as a conventional mouse and to evaluate the performance of the proposed device when operated by quadriplegic users. The device consisted of a video camera, computer software, and a target attached to the front part of a cap, which was placed on the user's head. The software captured images of the target coming from the video camera and processed them with the aim of determining the displacement from the center of the target and correlating this with the movement of the computer cursor. Evaluation of the interaction between each user and the proposed device was carried out using 24 multidirectional tests with two degrees of difficulty. Tested on 10 individuals with cervical spinal cord injury the developed pointing device adequately emulates the movement functions of the computer cursor. It is easy to use and can be learned quickly when operated by quadriplegic individuals.

Regueiro et al. studied a possible correlation between the BODE Index and variables assessed during the Activities of Daily Living assessment, performance on lower limber tests, and peripheral muscle impairment of the upper limb in 10 patients with chronic obstructive pulmonary disease, ranging from moderate to very severe obstruction, and found no correlation between the BODE Index and the ventilatory and metabolic responses in the Activities of Daily Living assessment. On the other hand, a correlation was observed between the BODE Index and the variables assessed in the 6MWTT, Sit-to-Stand Test, and Hand Grip Strength Test in moderate to very severe Chronic Obstructive Pulmonary Disease patients. This suggests that these tests can be employed as predictors of physical exercise capacity, perhaps as complementary tests to the BODE Index.

Turker et al. compared the landmark-guided technique versus the ultrasound-guided technique for internal jugular vein cannulation in 380 spontaneously breathing patients between April and November, 2008. Failed catheter placement, risk of complications from placement, risk of failure on first attempt at placement, number of attempts until successful catheterization, time to successful catheterization and the demographics of each patient were recorded. They found that internal jugular vein catheterization guided by real-time ultrasound results in a lower access time and a lower rate of immediate complications.

Potu et al. evaluated the effects of the petroleum ether extract of Cissus quadrangularis on the proliferation rate of bone marrow mesenchymal stem cells, the differentiation of marrow mesenchymal stem cells into osteoblasts (osteoblastogenesis) and extracellular matrix calcification and suggest that Cissus quadrangularis stimulates osteoblastogenesis and can be used as preventive/alternative natural medicine for bone diseases such as osteoporosis.

We also publish reviews on mortality in anesthesia, on recent trends in Brazilian medical research, and the guidelines on the management of human infection with the novel virus influenza A (H1N1) from the Hospital Das Clínicas of São Paulo University Medical College. We also include 3 case reports 